\title{
Family violence and its psychological management at the Emergency Department: A review
}

\author{
Pasquale Caponnetto,, 1 Marilena Maglia, ${ }^{1}$ Loredana Pistritto, ${ }^{1}$ Salvatore Ferlito, ${ }^{1}$ \\ Maria Concetta Cannella ${ }^{3}$
}

${ }^{1}$ University of Catania, Italy; ${ }^{2}$ University of Stirling, UK; ${ }^{3}$ ASP 3 Catania, Italy

\begin{abstract}
An Emergency Department provides emergency diagnosis and interventions, first clinical instrumental and laboratory examinations, actions needed to stabilize the patient. The purpose of this review was to evaluate the state-of-the-science focused on intimate partner violence. The review search period was from 2000 to 2019. The search was not restricted by language or geographical region, and was carried out by combining an exhaustive list of terms denoting intimate partner violence, domestic violence, emergency department care of women, women victims of violence, identifying abuse among women. Databases searched included MEDLINE, PsycINFO, Web of Science, Scopus, and Cochrane Library.
\end{abstract}

\section{Introduction}

For World Health Organization (WHO), violence is: "The intentional use of physical force or power, threatened or actual, again stone self, or against a group or community that either results in or has a high likelihood of resulting in injury, death, psychological harm, maldevelopment or deprivation."

Type of violence as follows.

Correspondence: Pasquale Caponnetto, Departement of Clinical and Experimental Biomedicine, University of Catania, Catania 95123, CT, Italy.

E-mail: pcapon@unict.it

Key words: Domestic violence, pink code, emergency department, intimate partner violence, identifying abuse.

Contributions: PC was the primary author of the manuscript. LP, MM, SF and MCC were engaged in the articles analysis. All authors contributed to the writing of the paper and approved it for pubblication.

Conflict of interest: The authors declare no potential conflict of interest. Funding: None.

Received for publication: 12 September 2019.

Accepted for publication: 28 October 2019.

This work is licensed under a Creative Commons AttributionNonCommercial 4.0 International License (CC BY-NC 4.0).

CC Copyright: the Author(s), 2019

Licensee PAGEPress, Italy

Health Psychology Research 2019; 7:8558

doi:10.4081/hpr.2019.8558
Sexual Violence: sexual harassment through threats, overwhelming or physical force use, sexual intercourse against will as well as being compelled to have sexual intercourse with others.

Physical Violence: aggression involving the use of physical force. This type of violence can include a wide range of ill-treatment that can go from a simple killer to the killing and can cause injury or severe physical damage. They are therefore included: slaps, beatings, thrusts, kicks, twisting of arms, bites, stabbing, strangulation, suffocation, burns and threats with an object or weapon.

Psychological Violence: It may include various types of behavior such as abandonment or ill treatment threats, homebased isolation, intimidation of children's removal, separation from the previous family and / or amateur network, offenses and insults even in public, verbal aggression and ongoing offenses, economic deprivations, obsessive surveillance of places or people attending the woman.

According to the latest WHO data, physical and sexual abuse is a health problem affecting a third of women in the world; The phenomenon is also progressively increasing in Italy and more women go to the Emergency Department after being subjected to violence. Intimate partner violence is one of the most common forms of violence against women and includes physical, sexual, and emotional abuse and controlling behaviours by an intimate partner (WHO, 2012).

Gender violence has many forms and affects women and men in a different way. Women are much more affected by violence. IPV is the most common form of violence against women and afflicts about $30 \%$ of women in the world (WHO, 2013).

According to the WHO, women in Africa are twice as likely to be IPVs compared to European women, and women from Southeast Asia are even less. Although the idea of a subdued and beaten wife is persistent and rooted in some cultures, it has been identified as a social problem in the Western world in the 1960s and was recognized as a health problem in the 1970s, defining violence against women in the 1980s Such as "the beaten woman's syndrome" as a clinical picture of severe, severe and repeated physical injuries to the woman. There are no official epidemiological studies on gender violence in Italy (Capucci et al., 2015), due to the lack of a national observatory on gender violence, the most recent data can then underestimate the phenomenon.

An ISTAT study of 2006 on a sample of women aged between 16 and 70 showed that 2,938,000 women were victims of physical or sexual violence by their partner, and $14.3 \%$ of women who currently have or have had a partner have at least been abused by their partner. In Italy, according to the latest data released by ISTAT in $2013,31.5 \%$ of women aged 16 to 70 (6 million 788 thousand), and thus twice as much as in 2006, suffered some form of physical or sexual violence during his life: $20.2 \%$ (4 million 353 thousand) suffered physical violence, $21 \%$ (4 million 520,000) sexual assault, 5.4\% (1 million 157 thousand), the most serious forms of sexual violence such as rape $(652,000)$ and attempted rape 
$(746,000)$. Women also suffer from many threats $(12.3 \%)$. They are often pushed or wrinkled (11.5\%), subject to slaps, kicks, punches and bites $(7.3 \%)$. Other times they get hit with objects that can hurt $(6.1 \%)$. Only $7.3 \%$ of cases of IPV are reported to the police. The percentage of cases reported is much higher in cases where women address emergency room, police, doctors or nurses.

It is important for Public Health to be able to accommodate and manage these situations, which require not only a clinical approach, but the humanization of healthcare while also ensuring the listening, recognition and implementation of a safety plan for the woman. The impact of IPV on health is vast.

Women undergoing IPV may have physical, sexual and reproductive problems, mental health problems and are at high risk of suffering from chronic conditions such as asthma and heart problems compared to un abused women (Black et al., 2011). The consequences of this problem are enormous, IPV is the leading cause of premature disability and premature death in women (IHME, 2013). "Global and Regional Estimates of Violence Against Women: Prevalence and Health Effects of Intimate Partners Violence and Non partner Sexual Violence", of World Health Organization (WHO, 2013) reported the following data: $35 \%$ of women in the world have at least once experienced an experience of physical and/or sexual violence; In most cases violence is committed by the partner. (In $30 \%$ of cases in the world) In the world, $38 \%$ of femicides are committed by the partner. Women who have been physically or sexually abused by their partners report higher rates than a number of major health problems, such as: being more likely to have abortion, depression and 1.5 more probabilities of having HIV.

\section{Materials and Methods}

The purpose of this review was to evaluate the state-of-the-science focused on intimate partner violence. The review search period was from 2000-2019. The search was not restricted by language or geographical region, and was carried out by combining an exhaustive list of terms denoting intimate partner violence, domestic violence, emergency department care of women, women victims of violence, identifying abuse among women. Databases searched included MEDLINE, PsycINFO, Web of Science, Scopus, and Cochrane Library.

\section{Characteristics of violence}

Violence, including physical ill-treatment, sexual and psychological abuse, can cause physical harm, forced social isolation, threats that let the victim live in a state of constant fear and create conflicts that do not allow the woman to receive the appropriate care. One third of the physical violence is visible but many women arriving at the emergency room often receive misdiagnosis and so the phenomenon of physical violence is underestimated because of the conflicting or missing information in the story told by patients. For these reasons there are guidelines that ED doctors and nurses should follow to recognize the signs of abuse. It should also be considered the potential harm in bringing to light such a delicate problem for a woman who in most cases is unwilling to voluntarily divulge the abuse she has suffered because he is psychologically unprepared and/or is not able to embark on a path that leads to the end of violence in his life. It's because of the potential damage to screening and the lack of evidence for an intervention that many reviews concluded that there is not enough evidence to regularly examine all women victims of violence. Routine screening, it would be useful to identify

those women who are considered high risk for IPV, such as women arriving at ED with multiple injuries, telling them to be abnormally procured. According to Reisenhofer et al. (2007) the risk factors of domestic violence are: female sex, aged 15 to 45, stories of child abuse, unemployed women, pregnant women, low social status, social isolation. Other risk factors are recent divorces or separations (many femicides committed by the partner occur immediately after the separation period).

Studies have reported results on demographic variables and the history of patients suffering from violence, but few have examined the relationship between IPV and physical "damage" among women who are in the ED. According to Wu et al. (2010) were identified the correlations between physical lesions and IPV. The lesions affecting the head-to-neck district are more likely than the lesions in the limbs and pelvis, the abdomen and chest. There are many difficulties to detect injuries, including the proximity of the aggressor during the story of the victims; the woman feels dependent on her partner, which can prevent her from telling the story, minimizing injuries, with the result that the relationship can lead to new violence and increasingly serious injuries.

\section{Physical damage in intimate partner violence}

It is important to be able to examine physical injuries to identify possible intimate partner violence (IPVs0 in women who are present in the ED. IPV lesions occur in specific regions of the body and so-called accidental injuries should be carefully examined. A systematic review of 2015 (Wu et al.) Does not identify isolated chest and abdomen lesions, upper and lower limbs as signs of IPV.

Instead, it is more likely to be in front of an IPV in multiple injury cases and in cases of lesions involving the face, neck, and head. About $70 \%$ of facial lesions observed in ED in women are secondary to IPV; The most common lesions are mandible fractures (in 39\% of cases). The injuries are, for example, distortions, fractures, dislocations, burns, lacerations, cut wounds, chest contractions.

Often women with facial, neck and head injuries have injuries to other parts of the body, such as distortions, fractures, discomforts, burns, tears, chest bruises. Insults on the abdomen, chest, and pelvis do not appear to be associated with IPV. IPV has serious and long-term consequences for the victims, and in addition to multiple physical injuries, persistent psychological disorders such as anxiety and depression, suicidal thoughts and suicidal attempts may persist. Women who do not exhibit acute symptoms but who have experienced an IPV are more frequently affected by chronic problems, like headaches and require painkillers more than women who have never been victims of violence. These factors can be controlled by identifying an IPV promptly. What happens in intimate relationships (beating, humiliation, rape and violence in general) reflects the perception of women as weak subjects who need to be controlled. In recent years, it's increased of the number of femicides, often resulting from a woman's decision to end a relationship. Spangaro et al. (2009) emphasize the complexity of the victim's relationship with his partner, and for women it can be very difficult to interrupt this relationship, because of the relationship of dependence on the partner, which can lead the woman to hide the state in which she is and continue the relationship of abuse she can cause ever more serious injuries. Many women who were then 
assassinated, have previously suffered many traumas and many IPV injuries, and these cases could be prevented. Men who feel inadequate, weak, insecure and with the awareness that their partner could base their choices on their own desires and aspirations, they think of overcoming these conflicts with the idea of the traditional physical superiority of men and their authority, using violence: men prefer to destroy the autonomy of women they come across rather than try to set aside an obsolete concept of masculinity and build a new male conscience.

\section{Attending to emergency department}

A Spanish study (Montero et al., 2011) analyses the effect of violence on the use of health services, including primary care, specialist services, SP access and hospital admissions (on a sample of 10815 women). International research focused primarily on partner violence, given its high prevalence and significant impact on health. Consequences of private violence are physical and mental problems.

Women with a history of violence use the health services more than women who have not been victims of violence. Violence is a problem for the healthcare system by increasing the overloaded costs, both in the short and long term; the consequences of violence are often chronic, such as reproductive problems, wounds, somatic disorders, drug abuse and high probability of being abused again. This study, conducted on women waiting for the ED, has been used to estimate the prevalence, risk factors and the health effects of women victims of violence. This study excluded illiterate women or serious disabilities, and women waiting for the ED with their partner. Of the 10815 women, $32.7 \%$ suffered violence during their life; $17.3 \%$ claim to have been abused by their partner; $10 \%$ who has been abused by other men.

Women who are victims of abuse often have low levels of education and low family income, have fewer social relationships, and often are divorced or divorced women. Women with IPV experience feelings of fear and shame, often associated with a sense of financial and emotional dependence, as well as the insecurity problems that condition their request for help. Many health professionals are not prepared to assist and help victims of violence, for this reason, women victims of abuse have a negative perception of health services and tend to minimize their abuses, especially when they are forced to receive healthcare if they are not ready to receive it. Health professionals need to be aware that many women may have been victims of IPV throughout their lives and that this violence had health effects that have not been limited to a single event, because abused women often seek health care without necessarily admit to having been victims of violence. It is therefore crucial to be able to identify cases of abuse. An Australian 2006 study states that many women are victims of IPV, are not identified and do not quite help when they seek ED assistance. The prevalence of the IPV phenomenon is underestimated because identification is often associated with the presence of visible physical lesions, but IPVs are not confined to this type of injury but also include psychological violence, sexual abuse, social isolation, economic restraint, intimidation, verbal threats. So, the physical aspect is obviously more easily measurable, but it must be considered that women who are victims of psychological abuse have deeper and hidden damages, as their self-esteem, their idea and their self-image is damaged, and physical healings that heal faster, psychological ones cause chronic and more easily conceived issues. The psychological consequences of IPV consist in having five times greater probabil- ity of suffering from depression or other psychological disorders; have five times more chance of suicide and are more likely to take psycho-drugs and alcohol, have unplanned pregnancies and abortions. The consequence of this state of suffering of women with IPV impels them to use health services more frequently both for physical and psychological problems. Unfortunately, it is often the case that although they turn more often to healthcare facilities and, in particular, despite being in the ED, often do not reveal that they are seeking help because they are victims of violence; For this reason, the ED operators must be able to recognize the victims of violence and to be able to afford the opportunity to trust themselves. Women who are victims of violence when they decide to trust and admit abuses seek help by going to the emergency room, addressing friends and family, psychologists, lawyers, and priests. First aid is the third source of help for women victims of violence (Reisenhofer et al., 2007).

The ED is the first contact point of these women with the health system. Emergency workers have unique and crucial role in identifying and treating women who seek help after being subjected to violence.

\section{Screening of intimate partner violence}

Recognizing IPV as a public health problem has led many health organizations to recommend screening and assistance procedures for women victims of IPV arriving at the ED. From the research (Klevens et al., 2012) on women victims of violence emerged the following data: the average age of women is 38 years, with a higher or lower education degree. The result of this screening study suggests providing ED practitioners with a list of risk factors and clinical signs to be considered in adult female patients to be used in EDs, which do not involve disadvantages in terms of overall health outcomes.

These findings provide important information for doctors and nurses, who are advised to use routine screening. Several studies suggest that there is inadequate screening in women victims of violence in first aid. ED's staff identify less than 5-10\% of violent women because they are treated for wounds or other symptoms they present, rather than being assessed as domestic violence symptoms. Because of the variety of symptoms among IPV-related women, practitioners are often not adequately prepared to identify and care for women with the consequences of an IPV. The most effective way to identify violent women is through screening, i.e. with direct requests from doctors and nurses who base their questions according to IPV risk factors. Women victims of violence awaiting the ED can fill out a routine screening questionnaire, nurses and security personnel can provide support. Early intervention can provide the opportunity to assist women with IPV and reduce the psychological, financial and social consequences experienced by the woman and her family. The literature provides a unambiguous answer to the acceptance of the screening questionnaire by women victims of IPV. Some women perceive screening as an invasion in their private sphere, intrusion into their privacy, leading to negative outcomes.

The New South Wales Department of Health (2005) has created routine screening to identify women victims of IPV by analysing early childhood risk factors and their mental health, alcohol/drug intake.

More studies are needed to explore the perceptions of women about violence and treatment received in the ED, explore how their perception of being taken care of influences their decision to stay 
in the ED and to trust the healthcare staff. Screening can be used to identify violence against women and to reduce violence or improve women's health. However, few studies have been conducted to evaluate patient outcomes after IPV identification and after intervention or counseling programs. Women victims of IPV and waiting for the ED feel a sense of general malaise, stunning, they feel private of their autonomy and they feel that they are being stigmatized and think they are even more isolated because they went to the ED, they feel the feeling of being misunderstood as they will be confident with ED operators, as they think that ED operators may be unharmed for their injuries and for the violence they have suffered. Yam (2000) examines issues related to the dissemination of violence and considers how these problems can be overcome thanks to routine screening. He found that both the questionnaire and the way ED staff approach the issue of IPV encourages or discourages the confession of being IPV victims. Yam (2000) identified that ED women who show IPV problems need to be treated with respect, dignity and compassion and when asked questions about IPV experience, this must be done in a private and secure environment so that you do not judge the woman .

\section{A new emergency code for domestic violence at Italian emergency department: pink code}

The E.D. can be a stressful and chaotic place and the nurses may not find the time to find out such a sensitive issue. This is why, in 2009, thanks to an agreement between the local health centre $n$. 9 and the Public Prosecutor office of Grosseto, the Code Pink has been created in Italy by Dr. Vittoria Doretti the Responsible Regional Network Code Pink, Tuscany Region, Italy. The Pink Code, is an approach geared to the acceptance of whoever suffers of abuse which starts inside the ED., in a unit where the experts shall visit and assist the patients. Thanks to the pioneering work of Dr. Vittoria Doretti, this model has gradually spread throughout the emergency services of Italian hospitals

In a Code Pink situation, an operational team, made of health professionals (doctors, nurses, psychologists) and law enforcement units, is being activate. Such team has to provide care and support to the victim, to open a formal investigation procedure to identify the aggressor and, if necessary, spread the alert to all the services nearby. The victim is given a Pink room, inside the E.D., where she will be supported by an operational team. Such room, which should not be identified for privacy reasons, is a sort of clinic which allows private visits and medical consultations, where the criminal forces units in charge of the investigation can have access only in some specific cases or if requested by the woman herself. The Room is provided with a specific exam kits, biological testing, photographic evidences, medical records, and IT supports accessible to the crime forces.

An evolved experiential model was developed and directed by Dr Maria Concetta Cannella at the hospital of Acireale-ASP 3 CT from 2016. In this case, however, the procedure was characterized for the timely activation of a psychologist and a social worker, available 24 hours a day, to the completion of the medical and nursing team (Cannella, 2018). What distinguished the model of Doctor Maria Concetta Cannella is the timely arrival of the psychologist and the social worker dedicated to the case within a maximum of 20-30 minutes from the call of the emergency department.

The Code Pink team is able, thanks to shared procedures and forces, (the local health centre, Law forces, anti-violence centre) to provide support and cares by respecting the victim privacy. The
E.D. Personnel may have few experiences or may not have a proper training on the impact that the IPV- Intimate Partner Violence might have on women, so it may not appreciate the need to evaluate the IPV. Such personnel may wrongly think that the IPV may happen only to low class women or they may not be ready to face the emotional and physical needs of abused women. The personnel are afraid of insulting or compromise the patients. The victims, on their side, don't reveal immediately the abuse because of the too busy or embarrassed personnel or because the author of the abuse is nearby during the visit. This is why the E.D. Personnel mush find a way to make the victims feel safe, cared and protected so to allow them to reveal any possible abuse. Nurses may give the questionnaire privately, use a simple language and assume a not judging attitude. It's important to figure out how to start a conversation with the patient about the violence, have a positive attitude and underline the confidentiality of the information. The E.D. Team has the chance to provide not only medical assistance but also to help and support the victim in such a sensitive case. The time spent in the E.D. waiting room is a very sensitive moment for the victim. Researches prove that women waiting at the E.D. due to injuries caused by their partners are the $1-3,5 \%$ of all the E.D users (Olive, 2017). Such numbers prove that of all the Intimate Partner Violence cases the E.D. Units see only the most serious cases of women abuses.

During the time spent in the E.D. waiting room, the personnel should investigate into women emotions and reactions. Women don't reveal the abuse for many reasons: the partner is still controlling them; they feel deeply ashamed, they are afraid of being judged, of being visited, and sometime for the presence of the social services in case of children protection. This is why the violence is not always or not immediately determined or identified by the E.D. Personnel because women consider the pro and cons of what happens if the reveal the abuse to the E.D. because in any case it will be an intrusion into their private life. Being the victim of an abuse and the consequent PTSD (post-traumatic stress disorder) symptoms shall be deeply analysed since it is hardly known the seriousness of the consequences of a IPV on women health (mental and physical) during the time spent in the waiting room. The main purpose of Olive's et al. 2016 research, based on a structured interview to IPV women, is to analyse their emotions and feelings soon after their partner abuse while waiting at the E.D. The interview, created for such study, pays attention to the participants' rights, to their comfort, protection and well-being; it lasts from 20 to 55 minutes and it is recorded and transcribed anonymously. The interview results aim at interpreting the experiences, the emotions and the sensations of IPV women waiting in a E.D. waiting room. The IPV forms were multiple and included emotional and psychological abuses, behaviour control, isolation, sexual abuse, physical violence, home isolation. The three key words, coming out from such inquiry, take into consideration the very first experiences, emotions and feelings of the IPV women during their first contact with the E.D. after their abuse: i) emotional response: "I don't want to be here"ii) "why did it happen to me?" iii) willingness to run away from ED The IPV women describe their emotional shock, their awareness to have their mind somewhere else, to feel on the line. They confess of being unable to think rationally and to live in a state of confusion and shock. These are the common symptoms of an acute stress as well as the anxiety, impaired concentration, the feeling of being detached and stupid. As soon as they arrive at the E.D. and after the triage, where they haven't been able to answer properly, they don't often understand the questions requested and go to the waiting room, where they don't want to wait because they are on edge, they feel anxious and they only 
want to hurry up and run away. This state of acute stress and frailty is made worse by the presence of many people nearby or in case of stereotyped questions. All the above-mentioned perceptions may represent an obstacle to the E.D. access and they may make the IPV women run away. Waiting at the E.D. and the experts and support cares for IPV women are key factors which have a strong impact on the E.D. outcomes. It is extremely important that a woman in such a sensitive situation may have the chance to relate to an expert in domestic violence. It is necessary for them to describe their own experiences immediately and to 11 relate to the right people. The IPV women hope to find, at the E.D. someone who completely understand them, who may dedicate his time to listen to their story, who may give a real support and help them to fight the awful feeling of isolation. What women feel after an abuse is similar to the acute stress perceived after a traumatic event and the consequences are either physical or psychological. The common symptoms of an acute stress reaction are: anxiety, low mood, emotional changes, sleeplessness, loneliness, recurring nightmares, the sensation of feeling stupid, detached, aggressive and reckless behaviours, palpitations, nausea, chest pain, headache, stomach pain, trouble breathing. The patients feel such symptoms during the abuse or immediately after and they may disappear quickly with no treatment, or may last for a long time. According to Forbes et al. (2014), the IPV victims have a very high probability to develop a PTSD disorder; this is why it's necessary to take a prompt action. The PTSD may lead to anxiety and depression (WHO, 2013). It's extremely important to provide the IPV women with a first psychological aid to prevent the development of any further consequences. It is also important to respect their willingness to talk about what happened, to identify their concerns and needs by encouraging the positive coping and by providing them with options and follow up. According to a Finnish study of 2013 the aggressors may suffer themselves for what they have done, by experiencing attitudes and psychological problems, guilt, depression, anxiety and shame and may fall in the use of drugs or lose their jobs. This is why the IPV is a multidimensional problem which requires a prompt action in order to cut the chain of violence or prevent it from occurring. There are also professionals, cultural barriers which obstacle a prompt action in case of abuses. Such barriers concern the lack of a proper preparation and skills of the medical staff, the stereotype of the "easy woman", who may have done something to deserve the abuse, personal behaviours, lack of time to look into a case of violence, lack of a H24 service, lack of a real protocol, a sense of frustration that nothing can be made. Being the object of an abuse or being a witness of a IPV may increase the feelings of anger, shame, depression and fear which may inhibit the treatment that the health staff may provide. A prompt action in the case of IPV may minimize the negative consequences. Women undergoing a screening for IPV are incline to talk about their experience; women may be screened as a routine procedure or in case of suspected violence. A quick action may be the turning point to break the chain of violence. It's a very critical issue which requires the cooperation of different forces in order to help the abused women and also the violent men. The victims of abuse are often unable to illustrate their situation at the E.D. and the 12 traumas caused by the IPV may affect the women ability at taking the right decisions. The E.D. can't count on a long-term service of IPV, this is why it's important the E.D. staff is being informed about the women support centre. The E.D. is often the first contact women have after an abuse and the staff is the first who has to evaluate and diagnose the woman situation and the family situation. It is also essential to cooperate with other experts in order to plan the immediate cares. The Finnish enquiry focuses on two issues: i) which kind of action does the E.D. staff usually take in case of women abuse?; 2) which attitude does the E.D. staff have towards to IPV?

The $25 \%$ of the 457 interviewed experts plan with the woman the action to be taken but the $45 \%$ of them does not often go deep into the cases of suspected IPV especially when the woman doesn't reveal spontaneously what happened. Only the $3 \%$ of them helps such women by giving them any kind of information regarding the way to stop the violence. The results of the mentioned enquiry reveal that the $50 \%$, approximately, of the E.D. staff does not follow any written procedure to handle the IPV. The lack of a protocol is to be considered as an obstacle in the intervention procedures; a specific written protocol and the chance to provide support represent the ways thanks to which the E.D. staff may help the IPV women and their family. According to the enquiry, a E.R which follows the written procedures to deal with the IPV is able to help women and men much more than the E.D. which does not follow such procedure. This is why a written protocol is highly recommended in each E.D. A good team work and the cooperation with other IPV centres are essential for the abuse prevention, to create an aid plan for abused women and violent men.

Undoubtedly, the physical injuries are a visible sign of the IPV and the health experts should be able to identify the IPV victims and to communicate the abuse to the relevant authorities. The IPV issue is underestimated, this is why many women arriving at the E.D. get the wrong diagnosis or sometimes they give conflicting versions of what happened. Such failure is basically due to the lack of a proper training of the health operators who are not able to recognize the signs of IPV. According to the American Medical Association the heath staff working in a E.D. must know and apply the protocol for the abuse diagnosis and care. What is missing is the approval of a valid routine IPV screening when the patient gets in the E.D. The lawyers and anti-violence centres agree on approving the validity of a routine screening since it may help the victim to get away from an abuse situation. IPV women should be informed about the consequences of the abuse allegation. They are often unprepared to interrupt the presence of violence in their lives. Despite not having a compulsory regulation 13 about that, women who are considered IPV patients are regularly screened thanks to the medical experts and to the anti-violence associations.

\section{References}

Black, M.C., Basile, K.C., Breiding, M.J., Smith, S.G., Walters, M.L., Merrick, M.T., Chen, J., \& Stevens, M.R (2011). The national intimate partner and sexual violence survey (NISVS): 2010 Summary Report. National Center for Injury Prevention and Control, Centers for Disease Control and Prevention, Atlanta, GA.

Capucci, R., Paganelli, C., Carboni, S., Cappadona, R., Marci, R., \& Rinaldi, G. (2015). Characteristics of Gender-Based Violence Determined from Emergency Room Visits. Violence and Gender, 2(2), 129-133.

Cannella, MC (2018). ASP CT, per le vittime di violenza, un percorso diagnostic terapeutico assistenziale integrato. Picologi\&Psicologia, n1, Dicembre.

Forbes, D., Lockwood, E., Phelps, A., Wade, D., Creamer, M., Bryant, R.A., McFarlane, A., Silove, D., Rees, S., Chapman, C., Slade, T., Mills, K., Teesson, M., \& O’Donnell M. (2014). Trauma at the hands of another: distinguishing PTSD patterns following intimate and nonintimate interpersonal and non 
interpersonal trauma in a nationally representative sample. Journal of Clinical Psychiatry, 75(2), 147-153.

Istituto Nazionale di Statistica ISTAT, (2014). La violenza contro le donne dentro e fuori la famiglia.

Istituto Nazionale di Statistica ISTAT, (2006). La violenza contro le donne, Indagine multiscopo sulle famiglie "Sicurezza delle donne".

Institute for Health Metrics and Evaluation, (2013). The Global Burden of Disease: Generating Evidence, Guiding Policy. Institute for Health Metrics and Evaluation, Seattle, WA.

Klevens, J., Kee, R., Trick, W., Garcia, D., Angulo, F., Jones, R., \& Sadowki. L. (2012). Effect of Screening for Partner Violence on Women's Quality of Life. Journal of the American Medical Association, 308(7), 681-689.

Leppakoski, T., \& Paavilainen, E. (2012). Interventions for women exposed to acute intimate partner violence: emergency professionals' perspective. Journal of Clinical Nursing, 22(15-16), 2273-2285.

Loke, A.Y., Wan Mei Lan, E., \& Hayter M. (2012). The lived experience of women victims of intimate partner violence. Journal of Clinical Nursing, 21(15-16), 2336-2346.

Montero, I., Ruiz-Perez, I., Martın-Baena, D., Talavera, M., Escriba-Aguir, V. \& Vives-Cases, C. (2011). Violence Against Women from Different Relationship Contexts and Health Care Utilization in Spain. Women's Health Issues, 21(5), 400-406.

NSW Health, (2005). Routine screening for 14 domestic violence program. Snapshot Report 2, NSW Department of Health, Sydney.

Olive, P. (2016). First contact: acute stress reactions and experiences of emergency department consultations following an incident of intimate partner violence. Journal of Clinical
Nursing, 26(15-16), 2317-2327.

Reisenhofer, S., \& Seibold, C. (2007). Emergency Department care of women experiencing intimate partner violence: Are we doing all we can? Contemporary Nurse, 24(1), 3-14.

Spangaro, J., Zwi, A.B., \& Poulos, R. (2009). The elusive search for definitive evidence on routine screening for intimate partner violence. Trauma, Violence \& Abuse, 10(1), 55-68.

Walton, M.A., Murray R., Cunningham, R.M., Chermack S.T., Barry, K.L., Booth, B.M., ..., Blow, F.C. (2009). Correlates of Intimate Partner Violence Among Men and Women in an Inner City Emergency Department. Journal of Addictive Diseases, 8(4), 366-381.

World Health Organization, (2012). Fact Sheet N 239 Violence against Women: Intimate Partner and Sexual Violence against Women. World Health Organisation, Geneva.

World Health Organization, (2013a). Global and Regional Estimates of Violence against Women: Prevalence and Health Effects of Intimate Partner Violence and Non-Partner Sexual Violence. World Health Organisation, Geneva.

World Health Organization, (2013b). Responding to Intimate Partner Violence and Sexual Violence against Women: WHO Clinical and Policy Guidelines. World Health Organisation, Geneva.

Wu, V., Huff, H., \& Bhandari, M. (2010). Pattern of Physical Injury Associated with Intimate Partner Violence in Women Presenting to the Emergency Department: A Systematic Review and Meta-Analysis. Trauma, violence and abuse, 11(2), 71-82.

Yam, M. (2000). Seen but not heard: Battered women's perceptions of the ED Experience. Journal of Emergency Nursing, 26(5), 464-470. 